ISSN 0258-7122

Bangladesh J. Agril. Res. 35(3) : 403-411, September 2010

\title{
VALIDATION OF DRAS MODEL FOR IRRIGATION OF WHEAT
}

\author{
P. K. SARKAR ${ }^{1}$, M. S. ISLAM ${ }^{2}$, S. K. BISWAS ${ }^{3}$ \\ M. A. HOSSAIN ${ }^{4}$ AND S. HASSAN ${ }^{5}$
}

\begin{abstract}
The study was conducted to validate the Drought Assessment (DRAS) model developed by the Center for Environmental and Geographic Information Services (CEGIS) for irrigation scheduling of wheat (variety: Shatabdi). The performance of the model was compared with the results obtained from the BARI recommended irrigation schedule. The field experiments were carried out during the years 2005-2006 through 2007-2008 in two agro-ecological zones. The locations were RARS, Jamalpur under agro-ecological zone 9 and farmers' field of FSR site, OFRD, Barind, Rajshahi under agro-ecological zone 26. Six different irrigation treatments including one rainfed with three replications were considered for the study. In respect of yield, BARI recommended irrigation practice performed better in Jamalpur (3.642 t/ha on average). Application of net irrigation requirement (NIR) as per DRAS model based on reported value yielded highest (3.598 t/ha on average) in the Barind area, Rajshahi. However, the yields from all irrigated treatments were very close to each other. From three years' study, the model performance was found quite satisfactory for irrigated wheat, especially in drought prone areas like Barind, Rajshahi. In respect of water productivity, the model performed almost similar to the BARI recommended practice in Jamalpur. It performed better in Barind region where irrigation water was used by the crop more efficiently.
\end{abstract}

Keywords: DRAS model, irrigation, wheat.

\section{Introduction}

To meet the requirement of cereal, wheat may be a viable substitute. It is highly responsive to irrigation water. With only 2 to 4 irrigations and through proper management, wheat yield can be increased by 50 to 100 percent. The irrigations at crown root initiation, maximum tillering and grain filling stages were found beneficial and also essential in increasing further grain yield of wheat (Rashid, 1994).

The month-wise distribution of rainfall in Bangladesh indicates that the wheat growing season (November-March) is the driest period of the year. The soil moisture is depleted rapidly in the later part of the crop if there is scanty or no rainfall during the wheat growing period. Soil moisture stress is reported to adversely affect the wheat yield (Rashid and Islam, 1986).

${ }^{1}$ Principal Scientific Officer, ${ }^{3}$ Scientific Officer, ${ }^{4}$ Senior Scientific Officer, ${ }^{5}$ Principal Scientific Officer and Head, IWM Division, Bangladesh Agricultural Research Institute (BARI), ${ }^{2}$ Chief Scientific Officer, FMPE Division, Bangladesh Agricultural Research Institute (BARI), Gazipur, Bangladesh. 
Poor supply of water and lack of proper irrigation scheduling are the main problems associated with the farmers (Saunders, 1991). Wheat generally suffers from water scarcity both in normal and dry years. Uneven distribution of rainfall throughout the year and water scarcity during the dry season affect the crop yield even in the average rainfall years. The drought could be managed efficiently through the adoption of well irrigation management practices. The DRAS model can be a useful tool to predict the net irrigation requirement (NIR) for wheat.

The model predicts the NIR on decade basis. When the crop suffers from water stress condition, the model prescribes an irrigation schedule for the crop. As the soil texture and type vary from field to field significantly, the model calculates the NIR for different land horizons. It also takes into account the soil characteristics and climatic conditions. Soil series-wise irrigation schedule is of great importance for the farmers for appropriate irrigation measures and this information can be disseminated through upazilla extension services.

Drought Assessment Model for wheat is a new concept in the traditional agriculture of Bangladesh. The framework of the Drought Assessment Model is described in details in BARC (2001). The DRAS model is developed by CEGIS (Centre for Environmental and Geographic Information Services) for predicting net irrigation requirement (NIR) and yield loss for different rice and non-rice crops on the basis of climatic, soil and cropping parameters. The CEGIS developed the model to predict the net irrigation requirement (NIR) and decided to validate it in the research as well as in the farmers' field. The present study was an attempt to validate the model for wheat crop. The specific objectives of the study were:

(i) to validate the model with field data at different locations;

(ii) to compare the model predicted NIR with the BARI recommended water needs for wheat cultivation;

(iii) to recommend the best practice for a specific location.

\section{Materials and Method}

Wheat (Triticum aestivum L.) cv. Shatabdi (BARI Gam 21) was used as the test crop for the study. It is a high yielding variety released by the Wheat Research Centre, BARI (WRC, 2003). It performs better even in some delayed planting. It is semi-heat tolerant and is suitable to cultivate after harvest of transplanted Aman rice and can be cultivated throughout the country.

The experiment was carried out during the rabi seasons of the years 2005-06 through 2007-08. The field studies were conducted at RARS farm, Jamalpur and farmer's field of FSR site, OFRD, Barind, Rajshahi. Jamalpur site falls under Agro-ecological Zone 9 (Old Brahmaputra Floodplain). The texture of the sub- 
soil is clay loam over and under lain by clay loam and loam, respectively. The field capacity of the soil is $30 \%$ and the bulk density is $1.45 \mathrm{gm} / \mathrm{cc}$. Rajshahi soil falls under Agro-ecological Zone 26 (High Barind Tract). The sub-soil texture is clay loam over and under lain by clay loam. The bulk density of the soil is 1.51 $\mathrm{gm} / \mathrm{cc}$ and the field capacity $31.5 \%$. The plot sizes were $4 \mathrm{~m} \times 5 \mathrm{~m}$ in Jamalpur and $3 \mathrm{~m} \times 3 \mathrm{~m}$ in Rajshahi. The experiment was arranged in a randomized complete block design (RCBD) with three replications. At all sites, fertilizers were applied as per recommended doses at the rate of $100,35,25$, and $22 \mathrm{~kg} / \mathrm{ha}$ of N, P, K, and S, respectively (BARI, 1999). Seeds were sown in second half of November during all the study years. The seed rate was $120 \mathrm{~kg} / \mathrm{ha}$. The crop was harvested in March of the cropping season. Soil moisture status in every plot was monitored by gravimetric method before each irrigation. BARI recommended irrigation treatment plots were irrigated upto field capacity of the soil and the other plots were irrigated as per recommendation of DRAS model. Yield and yield contributing parameters viz. plant height, spike length, grains per spike, 1000-grain weight, and grain yield were recorded.

The following treatments were used in the study;

$\mathrm{T}_{1}=$ No irrigation .

$\mathrm{T}_{2}=$ Application of irrigation based on BARI recommended practice (irrigation at 21, 45, and 65 days after sowing).

$\mathrm{T}_{3}=$ Application of net irrigation requirement (NIR) as per DRAS model based on reported value.

$\mathrm{T}_{4}=$ Application of NIR as per DRAS model based on actual field data .

$\mathrm{T}_{5}=$ Application of cumulative NIR as per DRAS model based on reported value.

$\mathrm{T}_{6}=$ Application of cumulative NIR as per DRAS model based on actual field data .

\section{Results and Discussion}

As mentioned earlier, the project was implemented in three rabi seasons of 20052006, 2006- 2007, and 2007-2008 years. But to avoid duplication of similar tables and discussion, only the achievements of 2007-2008 have been discussed in details in Table 1-6. Average results of three study years are also incorporated and discussed in Table 7-9.

The results obtained from the field study conducted during 2007-08 season at two sites are presented in Table 1 to 4 . Table 1 contains the yield and yield contributing parameters of different treatments of Jamalpur site. As seen from Table 1, major agronomic parameters like plant height, spike length, and number of grains per spike were influenced significantly by different irrigation regimes. However, 1000-grain weight did not show any significant difference among 
Table 1. Yield and yield contributing characters of wheat in different treatments during 2007-2008 at Jamalpur site.

\begin{tabular}{l|l|l|l|l|l|l|l}
\hline Treatment & $\begin{array}{c}\text { Plant } \\
\text { height } \\
(\mathrm{mm})\end{array}$ & $\begin{array}{c}\text { No. of } \\
\text { spikes/ } \\
\mathrm{m}^{2}\end{array}$ & $\begin{array}{c}\text { Spike } \\
\text { length } \\
(\mathrm{mm})\end{array}$ & $\begin{array}{c}\text { No. of } \\
\text { grains/ } \\
\text { spike }\end{array}$ & $\begin{array}{c}1000- \\
\text { grain wt } \\
(\mathrm{g})\end{array}$ & $\begin{array}{c}\text { Grain } \\
\text { yield } \\
(\mathrm{t} / \mathrm{ha})\end{array}$ & $\begin{array}{c}\text { Yield increase } \\
\text { over } \mathrm{T}_{2}(\%)\end{array}$ \\
\hline $\mathrm{T}_{1}$ & $842 \mathrm{c}$ & 284 & $72.3 \mathrm{~b}$ & $25.9 \mathrm{~d}$ & 44.5 & $\mathbf{2 . 9 1 7 c}$ & -29.15 \\
$\mathrm{~T}_{2}$ & $952 \mathrm{a}$ & 307 & $87.7 \mathrm{a}$ & $34.7 \mathrm{ab}$ & 46.5 & $\mathbf{4 . 1 1 7 a}$ & - \\
$\mathrm{T}_{3}$ & $936 \mathrm{ab}$ & 302 & $85.7 \mathrm{a}$ & $35.0 \mathrm{a}$ & 45.4 & $3.983 \mathrm{ab}$ & -3.25 \\
$\mathrm{~T}_{4}$ & $966 \mathrm{~b}$ & 325 & $82.7 \mathrm{a}$ & $33.3 \mathrm{abc}$ & 46.3 & $3.742 \mathrm{~b}$ & -9.11 \\
$\mathrm{~T}_{5}$ & $945 \mathrm{ab}$ & 298 & $82.3 \mathrm{a}$ & $31.6 \mathrm{c}$ & 47.0 & $3.808 \mathrm{~b}$ & -7.51 \\
$\mathrm{~T}_{6}$ & $932 \mathrm{ab}$ & 307 & $85.3 \mathrm{a}$ & $32.7 \mathrm{bc}$ & 46.7 & $3.933 \mathrm{ab}$ & -4.47 \\
\hline $\mathrm{CV}(\%)$ & 1.78 & 4.62 & 5.98 & 3.72 & 4.18 & 4.44 & - \\
LSD & 33.24 & NS & 8.88 & 2.18 & $\mathrm{NS}$ & 0.304 & - \\
\hline
\end{tabular}

the treatments. This result indicates that irrigation did not affect the size of the grains very much. In respect of grain yield, treatment $\mathrm{T}_{2}$ (BARI recommended practice) performed better (4.117 t/ha) in Jamalpur. However, the yield decrease of the model based treatments from that of BARI recommended practice was very low (less than 10 percent). The rainfed treatment produced the lowest yield (2.917 t/ha) and differed significantly from irrigated treatments. Table 2 shows the effect of irrigation on yield and yield contributing parameters of the crop at Rajshahi site. A distinct effect of irrigation on plant height, spikes/ $\mathrm{m}^{2}$, grains/spike and yield was observed. The trend of yield is somewhat different from that observed in Jamalpur. Treatment $\mathrm{T}_{4}$, produced the highest yield (4.153 t/ha) in Rajshahi site although no significant difference was observed among the irrigated treatments.

Table 2. Yield and yield contributing characters of wheat in different treatments during 2007- 2008 at Rajshahi site

\begin{tabular}{l|c|c|c|c|c|c|c}
\hline Treatment & $\begin{array}{c}\text { Plant } \\
\text { height } \\
(\mathrm{mm})\end{array}$ & $\begin{array}{c}\text { No. of } \\
\text { spikes/ } \\
\mathrm{m}^{2}\end{array}$ & $\begin{array}{c}\text { Spike } \\
\text { length } \\
(\mathrm{mm})\end{array}$ & $\begin{array}{c}\text { No. of } \\
\text { grains/ } \\
\text { spike }\end{array}$ & $\begin{array}{c}\text { 1000-grain } \\
\text { wt (g) }\end{array}$ & $\begin{array}{c}\text { Grain } \\
\text { yield } \\
\text { (t/ha) }\end{array}$ & $\begin{array}{c}\text { Yield } \\
\text { increase } \\
\text { over } \mathrm{T}_{2} \\
(\%)\end{array}$ \\
\hline $\mathrm{T}_{1}$ & $922 \mathrm{c}$ & $238 \mathrm{~b}$ & $95 \mathrm{c}$ & $35.1 \mathrm{c}$ & 45.1 & $2.389 \mathrm{~b}$ & -37.07 \\
$\mathrm{~T}_{2}$ & $970 \mathrm{ab}$ & $304 \mathrm{a}$ & $103 \mathrm{ab}$ & $43.7 \mathrm{a}$ & 45.3 & $3.803 \mathrm{a}$ & - \\
$\mathrm{T}_{3}$ & $1000 \mathrm{a}$ & $312 \mathrm{a}$ & $100 \mathrm{~b}$ & $41.2 \mathrm{~b}$ & 46.3 & $3.833 \mathrm{a}$ & 0.79 \\
$\mathrm{~T}_{4}$ & $995 \mathrm{a}$ & $317 \mathrm{a}$ & $106 \mathrm{a}$ & $44.6 \mathrm{a}$ & 45.7 & $\mathbf{4 . 1 5 3 a}$ & 9.20 \\
$\mathrm{~T}_{5}$ & $983 \mathrm{ab}$ & $290 \mathrm{a}$ & $107 \mathrm{a}$ & $45.5 \mathrm{a}$ & 46.6 & $3.930 \mathrm{a}$ & 3.34 \\
$\mathrm{~T}_{6}$ & $967 \mathrm{a}$ & $296 \mathrm{a}$ & $106 \mathrm{a}$ & $45.8 \mathrm{a}$ & 46.0 & $3.861 \mathrm{a}$ & 1.53 \\
\hline CV (\%) & 1.32 & 4.73 & 2.64 & 3.65 & 2.23 & 9.05 & \\
LSD & 23.4 & 11.6 & 4.95 & 2.84 & $\mathrm{NS}$ & 0.625 & \\
\hline
\end{tabular}


Table 3 shows the water use and water productivity of the treatments at Jamalpur site. It should be noted here that $\mathrm{T}_{3}$ and $\mathrm{T}_{4}$ received the highest number of irrigations (10 irrigations each). So, the least amount of drought was imposed on those treatments. But $\mathrm{T}_{4}$ produced lower yield (3.742 t/ha) than $\mathrm{T}_{3}$ (3.983 $\mathrm{t} / \mathrm{ha})$. However, higher water was used by $\mathrm{T}_{3}(330 \mathrm{~mm})$ than $\mathrm{T}_{4}(295 \mathrm{~mm})$. It should be noted that both the treatments $T_{5}$ and $T_{6}$ received the same number of irrigations (4 irrigations each) although they used different amount of water. Table 3 reveals that lesser the water use, higher the productivity, and it is the highest in treatment $\mathrm{T}_{1}$ (rainfed).

Table 3. Total water use (TWU) and water productivity (WP) of different treatments during 2007-2008 at Jamalpur site.

\begin{tabular}{l|l|l|l|l|l|l|l}
\hline Treatment & $\begin{array}{c}\text { No. of } \\
\text { irrigation }\end{array}$ & $\begin{array}{c}\text { Irrigation } \\
\text { water } \\
(\mathrm{mm})\end{array}$ & $\begin{array}{c}\text { Effective } \\
\text { rain fall } \\
(\mathrm{mm})\end{array}$ & $\begin{array}{c}\text { Soil } \\
\text { moisture } \\
\text { deficit } \\
(\mathrm{mm})\end{array}$ & $\begin{array}{c}\text { Total } \\
\text { water } \\
\text { use } \\
(\mathrm{mm})\end{array}$ & $\begin{array}{c}\text { Yield } \\
(\mathrm{t} / \mathrm{ha})\end{array}$ & $\begin{array}{c}\text { Water } \\
\text { productivity } \\
\left(\mathrm{kg} / \mathrm{m}^{3}\right)\end{array}$ \\
\hline $\mathrm{T}_{1}$ & 0 & 0 & $\mathbf{8 3}$ & 59 & $\mathbf{1 5 9}$ & 2.917 & 1.831 \\
$\mathrm{~T}_{2}$ & 3 & 140 & 83 & 43 & 291 & 4.117 & 1.415 \\
$\mathrm{~T}_{3}$ & 10 & 182 & 83 & 54 & 330 & 3.983 & 1.207 \\
$\mathrm{~T}_{4}$ & 10 & 144 & 83 & 29 & 295 & 3.742 & 1.269 \\
$\mathrm{~T}_{5}$ & 4 & 195 & 83 & 34 & $\mathbf{3 3 6}$ & 3.808 & 1.133 \\
$\mathrm{~T}_{6}$ & 4 & 154 & 83 & 50 & 310 & 3.933 & 1.269 \\
\hline
\end{tabular}

Table 4 shows the water use and water productivity of the treatments at Rajshahi site. Since there was a reasonable amount of rainfall $(83 \mathrm{~mm}$ in Jamalpur and 60 $\mathrm{mm}$ in Rajshahi) during the cropping season, the rainfed treatment produced comparatively high yields (2.917 t/ha at Jamalpur and 2.389 t/ha in Rajshahi) during 2007-2008 cropping season. The rainfed treatment showed the highest water productivity at both Rajshahi and Jamalpur sites (Table 3 and 4).

Table 4. Total water used and water productivity of different treatments during 2007-2008 at Rajshahi site.

\begin{tabular}{l|l|c|c|c|c|c|c}
\hline Treatment & $\begin{array}{c}\text { No. of } \\
\text { irrigation }\end{array}$ & $\begin{array}{c}\text { Amount of } \\
\text { irrigation } \\
\text { applied } \\
(\mathrm{mm})\end{array}$ & $\begin{array}{c}\text { Effective } \\
\text { rain fall } \\
(\mathrm{mm})\end{array}$ & $\begin{array}{c}\text { Soil } \\
\text { moisture } \\
\text { deficit } \\
(\mathrm{mm})\end{array}$ & $\begin{array}{c}\text { Total } \\
\text { water } \\
\text { use } \\
(\mathrm{mm})\end{array}$ & $\begin{array}{c}\text { Yield } \\
(\mathrm{t} / \mathrm{ha})\end{array}$ & $\begin{array}{c}\text { Water } \\
\text { productivity } \\
\left(\mathrm{kg} / \mathrm{m}^{3}\right)\end{array}$ \\
\hline $\mathrm{T}_{1}$ & 0 & 0 & 60 & 52 & 112 & 2.389 & 2.13 \\
$\mathrm{~T}_{2}$ & 3 & 192 & 60 & 36 & 288 & 3.803 & 1.33 \\
$\mathrm{~T}_{3}$ & 10 & 216 & 60 & 47 & 312 & 3.833 & 1.25 \\
$\mathrm{~T}_{4}$ & 10 & 184 & 60 & 22 & 276 & 4.153 & 1.51 \\
$\mathrm{~T}_{5}$ & 4 & 216 & 60 & 27 & 303 & 3.930 & 1.30 \\
$\mathrm{~T}_{6}$ & 4 & 184 & 60 & 43 & 287 & 3.861 & 1.35 \\
\hline
\end{tabular}


Economic analysis was done for both the study sites. The results are summarized in Table 5 and 6 . All variable cost items were considered as per the market values to estimate the total variable cost (TVC) for different treatments. Wheat cultivation using BARI recommended practice (treatment $\mathrm{T}_{2}$ ) was found

Table 5. Comparative cost and return from different treatments in Jamalpur during 2007- 2008.

\begin{tabular}{l|l|l|l|l|l|l}
\hline \multirow{2}{*}{ Item } & \multicolumn{6}{c}{ Different irrigation sequences } \\
\cline { 2 - 8 } & $\begin{array}{c}\mathrm{T}_{1} \\
(\mathrm{Tk} / \mathrm{ha})\end{array}$ & $\begin{array}{c}\mathrm{T}_{2} \\
(\mathrm{Tk} / \mathrm{ha})\end{array}$ & $\begin{array}{c}\mathrm{T}_{3} \\
(\mathrm{Tk} / \mathrm{ha})\end{array}$ & $\begin{array}{c}\mathrm{T}_{4} \\
(\mathrm{Tk} / \mathrm{ha})\end{array}$ & $\begin{array}{c}\mathrm{T}_{5} \\
(\mathrm{Tk} / \mathrm{ha})\end{array}$ & $\begin{array}{c}\mathrm{T}_{6} \\
(\mathrm{Tk} / \mathrm{ha})\end{array}$ \\
\hline $\begin{array}{l}\text { Human Labour } \\
\text { Land } \\
\text { preparation }\end{array}$ & 12240 & 12600 & 12720 & 12720 & 13440 & 13440 \\
Seeding & 3000 & 3000 & 3000 & 3000 & 3000 & 3000 \\
Manure & 4950 & 4950 & 4950 & 4950 & 4950 & 4950 \\
\hline Fertilizers & 8000 & 8000 & 8000 & 8000 & 8000 & 8000 \\
\hline Urea & 1356 & 1356 & 1356 & 1356 & 1356 & 1356 \\
TSP & 3816 & 3816 & 3816 & 3816 & 3816 & 3816 \\
MP & 2368 & 2368 & 2368 & 2368 & 2368 & 2368 \\
Gypsham & 420 & 420 & 420 & 420 & 420 & 420 \\
Irrigation & - & 2805 & 3094 & 2550 & 3315 & 2616 \\
Total variable cost & 36150 & 39315 & 39724 & 39180 & 40665 & 39968 \\
Gross return & 93344 & 131744 & 127456 & 119744 & 121856 & 125856 \\
Gross margin & 57194 & 92429 & 87732 & 80564 & 81191 & 85888 \\
BCR & 2.58 & 3.35 & 3.21 & 3.06 & 3.00 & 3.15 \\
\hline
\end{tabular}

Table 6. Comparative cost and return from different treatments in Rajshahi during 2007-08.

\begin{tabular}{l|l|l|l|l|l|l}
\hline \multirow{2}{*}{\multicolumn{1}{c}{ Item }} & \multicolumn{6}{c}{ Different irrigation sequences } \\
\cline { 2 - 8 } & $\begin{array}{c}\mathrm{T}_{1} \\
(\mathrm{Tk} / \mathrm{ha})\end{array}$ & $\begin{array}{c}\mathrm{T}_{2} \\
(\mathrm{Tk} / \mathrm{ha})\end{array}$ & $\begin{array}{c}\mathrm{T}_{3} \\
(\mathrm{Tk} / \mathrm{ha})\end{array}$ & $\begin{array}{c}\mathrm{T}_{4} \\
(\mathrm{Tk} / \mathrm{ha})\end{array}$ & $\begin{array}{c}\mathrm{T}_{5} \\
(\mathrm{Tk} / \mathrm{ha})\end{array}$ & $\begin{array}{c}\mathrm{T}_{6} \\
(\mathrm{Tk} / \mathrm{ha})\end{array}$ \\
\hline Human Labour & 12240 & 12600 & 12720 & 12720 & 13440 & 13440 \\
Land preparation & 3000 & 3000 & 3000 & 3000 & 3000 & 3000 \\
Seeding & 4950 & 4950 & 4950 & 4950 & 4950 & 4950 \\
Manure & 8000 & 8000 & 8000 & 8000 & 8000 & 8000 \\
\hline Fertilizers & 1356 & 1356 & 1356 & 1356 & 1356 & 1356 \\
\hline Urea & 3816 & 3816 & 3816 & 3816 & 3816 & 3816 \\
TSP & 2368 & 2368 & 2368 & 2368 & 2368 & 2368 \\
MP & 420 & 420 & 420 & 420 & 420 & 420 \\
Gypsham & - & 3264 & 3672 & 3128 & 3672 & 3128 \\
Irrigation & 36150 & 39774 & 40302 & 39758 & 41022 & 40478 \\
Total variable cost & 77404 & 125500 & 126490 & 137049 & 129690 & 127413 \\
Gross return & 41254 & 85726 & 86188 & 97291 & 88668 & 86935 \\
Gross margin & 2.14 & 3.16 & 3.14 & 3.45 & 3.16 & 3.15 \\
BCR & \multicolumn{7}{c}{}
\end{tabular}


comparatively profitable (highest BCR, 3.35) than the model based practices in Jamalpur. However, a model based treatment $\mathrm{T}_{4}$ (application of NIR as per DRAS model based on actual field data) performed best in respect of profitability (highest BCR. 3.45) in Barind, Rajshahi.

\section{Three years' combined results and discussion}

The study was conducted during three consecutive years from 2005-2006 to 2007-2008. Grain yield and total water use (TWU) by different treatments during three study years at two locations are presented in Table 7 and 8. Average grain yield and TWU along with water productivity (WP) are also shown in the same tables. Some variations in the performances of the treatments were observed in two locations. Treatment $\mathrm{T}_{2}$ (BARI recommended irrigation practice) yielded highest (3.648 t/ha on average) at Jamalpur site (Table 7), but the treatments based on the DRAS model $\left(T_{3}, T_{3}, T_{5}\right.$, and $\left.T_{6}\right)$ performed better at Rajshahi site (Table 8). However, the yield difference among the irrigated treatments was very low (below 10\%). In respect of crop water use, no distinct variation was observed among the irrigated treatments. At both the study sites, the treatment $T_{4}$ (application of NIR as per DRAS model based on actual field data) and treatment $\mathrm{T}_{6}$ (application of cumulative NIR as per DRAS model based on actual field data) used irrigation water more efficiently than the other irrigated treatments. So, the water productivity for these treatments was comparatively high (Table 7 and 8).

Table 7. Yield, total water use and water productivity of different treatments at Jamalpur site.

\begin{tabular}{|c|c|c|c|c|c|c|c|c|c|}
\hline \multirow{2}{*}{ Treatment } & \multicolumn{2}{|c|}{$\begin{array}{c}\text { Year } \\
2005-2006\end{array}$} & \multicolumn{2}{|c|}{$\begin{array}{c}\text { Year } \\
2006-2007\end{array}$} & \multicolumn{2}{|c|}{$\begin{array}{c}\text { Year } \\
2007-2008 \\
\end{array}$} & \multirow{2}{*}{$\begin{array}{l}\text { Average } \\
\text { yield } \\
\text { (t/ha) }\end{array}$} & \multirow{2}{*}{$\begin{array}{l}\text { Average } \\
\text { TWU } \\
(\mathrm{mm})\end{array}$} & \multirow{2}{*}{$\begin{array}{c}\text { Water } \\
\text { productivity } \\
\left(\mathrm{kg} / \mathrm{m}^{3}\right)\end{array}$} \\
\hline & \begin{tabular}{|l} 
Yield \\
(t/ha)
\end{tabular} & $\begin{array}{l}\text { TWU } \\
(\mathrm{mm})\end{array}$ & \begin{tabular}{|l} 
Yield \\
(t/ha)
\end{tabular} & $\begin{array}{l}\text { TWU } \\
(\mathrm{mm})\end{array}$ & \begin{tabular}{|l} 
Yield \\
(t/ha)
\end{tabular} & $\begin{array}{l}\text { TWU } \\
(\mathrm{mm})\end{array}$ & & & \\
\hline 1 & 1.652 & 88 & 1.989 & 122 & 2.917 & 159 & 2.100 & 123 & 1.92 \\
\hline$\Gamma_{2}$ & 3.685 & 238 & 3.142 & 340 & 4.117 & 291 & 3.648 & 290 & 1.34 \\
\hline$\Gamma_{3}$ & 3.415 & 208 & 2.234 & 347 & 3.983 & 330 & 3.544 & 295 & 1.20 \\
\hline $\mathrm{T}_{4}$ & 3.320 & 203 & 3.399 & 272 & 3.742 & 293 & 3.487 & 256 & 1.36 \\
\hline $\mathrm{T}_{5}$ & 3.569 & 231 & 3.030 & 319 & 3.080 & 336 & 3.469 & 295 & 1.24 \\
\hline - & 3.478 & 190 & 3.233 & 283 & 3.933 & 310 & 3.548 & 261 & 1.36 \\
\hline
\end{tabular}

The rainfed treatment performed best in respect of water productivity. This was due to the fact that this treatment used $42 \%$ water over that produced the highest yield at Jamalpur. Even it produced about $60 \%$ grain of the highest yielder. This indicates that no irrigated treatment used some residual soil moisture and rainfall occurred during the three cropping seasons. This provided a little scope for the plants to overcome an acute shortage of water. As a result, a considerable yield of 
$2.186 \mathrm{t} / \mathrm{ha}$ was produced by the treatment giving the highest water productivity of $1.92 \mathrm{~kg} / \mathrm{m}^{3}$. However, this output is not considerable in the context of increasing food production and land use productivity. The model performed better than the BARI traditional irrigation practice in respect of both yield and water use in Barind area, Rajshahi.

Table 8. Yield, total water use and water productivity of different treatments in Rajshahi site.

\begin{tabular}{|c|c|c|c|c|c|c|c|c|c|}
\hline \multirow{2}{*}{ Treatment } & \multicolumn{2}{|c|}{$\begin{array}{c}\text { Year } \\
\text { 2005-2006 } \\
\end{array}$} & \multicolumn{2}{|c|}{$\begin{array}{c}\text { Year } \\
\text { 2006-2007 }\end{array}$} & \multicolumn{2}{|c|}{$\begin{array}{c}\text { Year } \\
\text { 2007-2008 } \\
\end{array}$} & \multirow{2}{*}{\begin{tabular}{|c|} 
Average \\
yield \\
(t/ha)
\end{tabular}} & \multirow{2}{*}{$\begin{array}{c}\text { Average } \\
\text { TWU } \\
(\mathrm{mm})\end{array}$} & \multirow{2}{*}{$\begin{array}{c}\text { Water } \\
\text { productivity } \\
\left(\mathrm{kg} / \mathrm{m}^{3}\right)\end{array}$} \\
\hline & $\begin{array}{c}\text { Yield } \\
(\mathrm{t} / \mathrm{ha})\end{array}$ & $\begin{array}{l}\text { TWU } \\
(\mathrm{mm})\end{array}$ & $\begin{array}{l}\text { Yield } \\
\text { (t/ha) }\end{array}$ & $\begin{array}{l}\text { TWU } \\
(\mathrm{mm})\end{array}$ & $\begin{array}{l}\text { Yield } \\
\text { (t/ha) }\end{array}$ & $\begin{array}{l}\text { TWU } \\
(\mathrm{mm})\end{array}$ & & & \\
\hline $\mathrm{T}_{1}$ & 1.599 & 91 & 1.37 & 91 & 2.389 & 112 & 1.786 & 98 & 1.82 \\
\hline $\mathrm{T}_{2}$ & 3.449 & 256 & 2.78 & 266 & 3.803 & 288 & 3.344 & 270 & 1.24 \\
\hline $\mathrm{T}_{3}$ & 3.651 & 244 & 3.31 & 311 & 3.833 & 312 & 3.598 & 289 & 1.25 \\
\hline $\mathrm{T}_{4}$ & 3.649 & 209 & 2.86 & 265 & 4.153 & 276 & 3.554 & 250 & 1.42 \\
\hline $\mathrm{T}_{5}$ & 3.549 & 244 & 3.15 & 260 & 3.930 & 303 & 3.543 & 269 & 1.32 \\
\hline $\mathrm{T}_{6}$ & 3.909 & 209 & 2.85 & 263 & 3.861 & 287 & 3.540 & 253 & 1.38 \\
\hline
\end{tabular}

A comparative result on grain yield and total water use at two locations are shown in Table 9.

Table 9. Comparative average yield and total water use of two locations (Three years' average).

\begin{tabular}{|c|c|c|c|c|}
\hline \multirow[b]{2}{*}{ Treatment } & \multicolumn{2}{|c|}{ Jamalpur } & \multicolumn{2}{|c|}{ Rajshahi } \\
\hline & $\begin{array}{l}\text { Yield } \\
\text { (t/ha) }\end{array}$ & $\begin{array}{c}\text { Total water use } \\
(\mathrm{mm})\end{array}$ & $\begin{array}{l}\text { Yield } \\
\text { (t/ha) }\end{array}$ & $\begin{array}{c}\text { Total water use } \\
\text { (mm) }\end{array}$ \\
\hline$\overline{\mathrm{T}_{1}}$ & 2.186 & 114 & 1.786 & 98 \\
\hline $\mathrm{T}_{2}$ & 3.642 & 271 & 3.344 & 270 \\
\hline $\mathrm{T}_{3}$ & 3.544 & 295 & 3.598 & 289 \\
\hline $\mathrm{T}_{4}$ & 3.487 & 256 & 3.554 & 250 \\
\hline $\mathrm{T}_{5}$ & 3.469 & 280 & 3.543 & 269 \\
\hline $\mathrm{T}_{6}$ & 3.548 & 261 & 3.540 & 253 \\
\hline
\end{tabular}

Highest average grain yield of $3.642 \mathrm{t} / \mathrm{ha}$ was obtained using less water (271 mm) at Jamalpur site. On the other hand, highest average grain yield of $3.598 \mathrm{t} / \mathrm{ha}$ was obtained from a model based treatment $T_{3}$ at Barind, Rajshahi site. However, treatment $\mathrm{T}_{3}$ used the maximum water (295 $\mathrm{mm}$ and $289 \mathrm{~mm}$, respectively) at both the sites. 


\section{Conclusion}

From the results, it is evident that DRAS model performs well in the drought prone locations as the plants can utilize the irrigated water in a better way. BARI recommended irrigation practice seems good for locations having adequate water holding capacity of soils and higher rainfall situation. However, the difference between other two systems in respect of yield being very low, DRAS model based irrigation scheduling may be advocated to wheat growing farmers of the country for its better water use capacity and water productivity

\section{References}

BARI. 1999. BARI Technology Handbook. Bangladesh Agricultural Research Institute, Gazipur.

BARC. 2001. Contract research project report. Application of agro-ecological zones database in drought management and water availability assessment. June 2001, BARC, Farmgate, Dhaka.

Rashid, M. H. 1994. Irrigation Information Package for Planning Irrigation for Diversified Crops. Canadian Executing Agency. Crop Diversification Program (CDP), Ministry of Agriculture, Bangladesh.

Rashid, M. H. and T. Islam. 1986. Assessment of Availability of Water for Wheat Cultivation Strategies for Effective Use. A review paper in the proceedings of the Third National Wheat Training Workshop, held on August 4-5, 1986. WRC, BARI, Joydebpur, Bangladesh.

Saunders, D. A. 1991. Report of an on-farm survey at Jessore and Kustia: Wheat farmers' practices and perceptions, WRC, BARI, Nashipur, Dinajpur. Monograph No. 8. 30p.

WRC. 2003. Shatabdi (BARI Gam 21). Wheat Research Centre, BARI, Dinajpur, Bangladesh (in Bangla). 